\title{
ОСНОВНАЯ СУБГАРМОНИЧЕСКАЯ СОСТАВЛЯЮЩАЯ УПРАВЛЯЕМОГО ВЫПРЯМИТЕЛЯ
}

\author{
(Представил И. Эпик)
}

При разработке качественных управляемых выпрямителей (УВ) большой мощности следует минимизировать амплитуду субгармонических составляющих, обусловленных несимметричностью сети. Для шестиимпульсного выпрямителя $(m=6)$ наибольшей из составляющих является первая с частотой $100 \Gamma u$, которая трудно подавляется в пассивных $(L, C)$-фильтрах. Ее некоторая минимизация достигается путем выбора рациональной системы импульсно-фазового управления (СИФУ). Однако влияние СИФУ на субгармонический состав не изучено еще достаточно исчерпывающе $\left[{ }^{1-4}\right]$.

Ниже приводятся результаты цифрового гармонического анализа для УВ с различными алгоритмами работы СИФУ. Относительная к напряжению прямой последовательности сети амплитуда $100 \Gamma u$ составляющей $U_{100} / U_{1}$ рассчитана приближенно с заменой интегралов в формулах Әйлера [ $\left.{ }^{5}\right]$ суммами при трапецеидальной аппроксимации интегрируемой функции. В качестве этой функции выбрана математическая модель (MМ) выпрямителя, управляемого различного типа СИФУ:

$$
\begin{gathered}
U_{d}(N) / U_{1}=\sum_{l=1}^{m} U_{\pi l}(N)=\sum_{l=1}^{m} L_{l}(\sin (N+\pi(m-(l-1)) / 3)+ \\
+\varepsilon \sin (N+\pi(l-1) / 3+\psi)),
\end{gathered}
$$

где $U_{л l}(N)$ - линейное напряжение сети, $U_{d}(N)$ - выходное напряжение УВ, $N \Leftrightarrow \omega t-$ цифровой аргумент (текущая фаза), $l \in\{1, \ldots, m\}-$ номер вентиля и линейного напряжения, $U_{1}$ и $U_{2}-$ амплитуды линейного напряжения прямой и обратной последовательностей соответственно, $\varepsilon=U_{2} / U_{1}-$ модуль (коэффициент) несимметричности, $\psi=$ $=\left\llcorner\dot{U}_{1} \dot{U}_{2}\right.$ - аргумент несимметричности, $L_{l} \in\{0,1\}-$ зависящая от алгоритма работы СИФУ и $U_{л l}(N)$ коммутационная функция, суть которой раскрывается ниже.

Функция (1) периодична по ансамблю ее аргументов

$$
U_{d}(N) \equiv U_{d}(N+180),
$$

что позволяет в некоторых случаях сократить $M M$ до $l \leqslant 3$. При записи (2) и в дальнейшем предполагается «градусная» дискретность $N$. При анализе приняты следующие упрощающие допущения: 1) вентили УВ являются идеальными полууправляемыми ключами; 2) коммутационный 


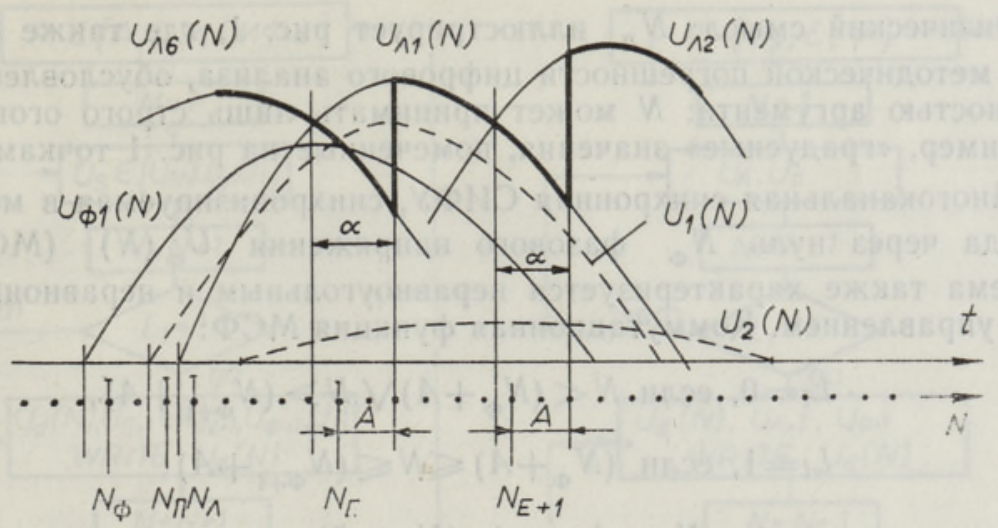

Рис. 1. Эпюра напряжения питания УВ и условия синхронизации СИФУ с линейным развертывающим сигналом.

процесс происходит мгновенно; 3) СИФУ лишена инструментальной погрешности, т. е. не вносит в несимметрию ММ своего вклада.

Гармоническому анализу подвергались УВ со следующими алгоритмами работы СИФУ:

1. Многоканальная синхронная СИФУ (рис. 1), синхронизируемая в момент естественной коммутации $N_{E}(\mathrm{MCE})$. Основным отличием $\mathrm{MCE}$ от остальных систем является инвариантность ее угла управления $\alpha \mathrm{K}$ несимметричности сети $\alpha_{l}=\alpha_{l+1} \neq f(\varepsilon, \psi)$. Коммутационная функция $\mathrm{MCE}$, входящая в (1), описывается условиями:

$$
\begin{gathered}
L_{l}=0, \text { если } N<\left(N_{E}+A\right) \bigvee N>\left(N_{E+1}+A\right), \\
L_{l}=1, \text { если }\left(N_{E}+A\right) \leqslant N \leqslant\left(N_{E+1}+A\right) ; \\
N_{E}=1 . \arcsin U_{л l-1}(N) \leqslant U_{л l}(N), \\
N_{E+\mathbf{r}}=1 . \arcsin U_{\text {лl }}(N) \leqslant U_{\text {лl+1 }}(N) .
\end{gathered}
$$

Принятая в (4) для $N_{E}$ и $N_{E+1}$ запись означает, что соответствующему неравенству удовлетворяет первый из числа аргументов $\{N\}$. Символ $A$ в (3) есть цифровой эквивалент угла управления $\alpha$, отсчитанного с момента синхронизации. Для MCE в пределах дискретности $A \Leftrightarrow \alpha$, для следующих синхронных СИФУ $A$ и $\alpha$ различны.

2. Многоканальная синхронная СИФУ, синхронизируемая в момент перехода через нуль $N_{\text {л }}$ линейного напряжения $U_{\text {л }}(N)$ (MСЛ). Эта СИФУ характеризуется неравноугольным $A_{l} \neq A_{l+1}$ и неравноннтервальным $N\left(\alpha_{l}\right)-N\left(\alpha_{l+1}\right)=\operatorname{var}$ управлением. Коммутационная функция МСЛ:

$$
\begin{gathered}
L_{t}=0, \text { если } N<\left(N_{л}+A\right) \vee N>\left(N_{л+1}+A\right), \\
L_{t}=1, \text { если }\left(N_{л}+A\right) \leqslant N \leqslant\left(N_{л+1}+A\right) ; \\
N_{л}=1 \cdot \arcsin \left(U_{л l}>0\right), \\
N_{\text {л+1 }}=1 \cdot \arcsin \left(U_{\text {лl+1 }}>0\right),
\end{gathered}
$$


Физический смысл $N_{л}$ иллюстрирует рис. 1, где также показана суть методической погрешности цифрового анализа, обусловленной дискретностью аргумента: $N$ может принимать лишь строго оговоренные, например, «градусные» значения, помеченные на рис. 1 точками.

3. Многоканальная синхронная СИФУ, синхронизируемая в момент перехода через нуль $N_{\Phi}$ фазового напряжения $U_{\Phi}(N)$ (МСФ). Эта система также характеризуется неравноугольным и неравноинтервальным управлением. Коммутационная функция МСФ:

$$
\begin{gathered}
L_{l}=0, \text { если } N<\left(N_{\Phi}+A\right) \vee N>\left(N_{\Phi+1}+A\right), \\
L_{l}=1, \text { если }\left(N_{\Phi}+A\right) \leqslant N \leqslant\left(N_{\Phi+1}+A\right) ; \\
N_{\Phi}=1 . \arcsin \left(U_{\Phi}>0\right), \\
N_{\Phi+1}=1 . \arcsin \left(U_{\Phi+1}>0\right) .
\end{gathered}
$$

4. Многоканальная синхронная СИФУ, синхронизируемая в момент перехода через нуль $N_{\text {п }}$ напряжения прямой последовательности $U_{1}(N)$ (МСП). Эта СИФУ не имеет пока технических реализаций, однако она хорошо описывает свойства достаточно известной одноканальной синхронной СИФУ. Ее интервалы $N\left(\alpha_{l}\right)-N\left(\alpha_{l+1}\right)=60$ инвариантны к $(\varepsilon, \psi)$. Коммутационная функция МСП:

$$
\begin{gathered}
L_{l}=0, \text { если } N_{<}\left(N_{\Pi}+A\right) \bigvee N>\left(N_{\Pi+1}+A\right), \\
L_{l}=1, \text { если }\left(N_{\Pi}+A\right) \leqslant N \leqslant\left(N_{\Pi+1}+A\right) ; \\
N_{\Pi}=1 . \arcsin U_{1}>0, \\
N_{\Pi+1}=1 . \arcsin \left(-a U_{1}\right)>0=N_{\Pi}+60 .
\end{gathered}
$$

Здесь $a=\mathrm{e}^{\mathrm{j} 2 \pi / 3}-$ оператор поворота.

Объединяющий рассмотренные СИФУ граф ММ с линейным развер. тывающим сигналом показан на рис. 2 , где приняты следующие обозначения: $P-$ варьируемый параметр, $U_{S}-$ напряжение синхронизации. Выходом $M M$ УВ является массив значений $U_{d}(N)$, обозначенный на графе символом WRITE.

5. Многоканальная арккосинусная СИФУ (МСА), развертывающий сигнал которой формируется от соответствующих фазовых напряжений их поворотом $U_{\mathrm{p}}=U_{\Phi} \mathrm{e}^{-j \pi / 2}$. Эта СИФУ не требует специального синхронизатора, определяющего момент синхронизации. Коммутационная функция МСA:

$$
\begin{aligned}
& L_{l}=0, \text { если }\left(U_{\mathrm{P}}<U_{\mathrm{y}}\right) \vee\left(U_{\mathrm{P}+1}>U_{\mathrm{y}}\right), \\
& L_{l}=1, \text { если }\left(U_{\mathrm{P}}>U_{\mathrm{y}}\right) \&\left(U_{\mathrm{P}+1}<U_{\mathrm{y}}\right) .
\end{aligned}
$$

Здесь $U_{y}$ аналоговый сигнал управления, задающий $\alpha$.

Из-за несимметричности сети в данной и последующих СИФу осуществляется только неравноугольное и неравноинтервальное управление, а заданием $U_{y}$ могут быть определены лишь средние значения $\alpha$. Граф ММ УВ с МСА показан на рис. 3. 


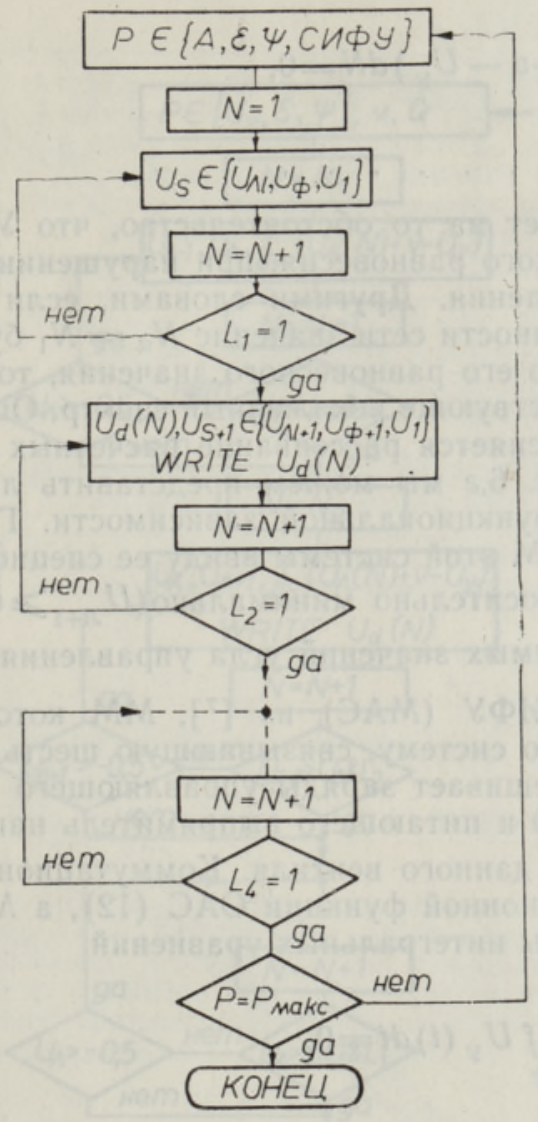

Рис. 2. Обобщенная ММ УВ, оснащенного синхронной СИФУ с линейным развертывающим сигналом (МСЕ, МСЛ, МСФ, МСП).

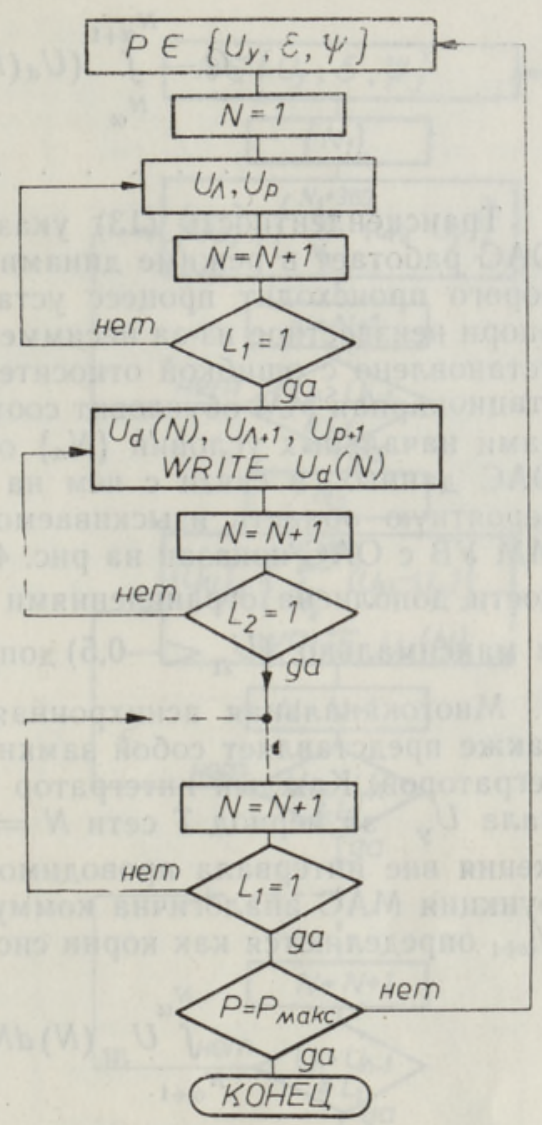

Рис. 3. ММ УВ, оснащенного арккосинусной СИФУ (МСА).

6. Одноканальная асинхронная СИФУ (ОАС). Она существенно отличается от вышеописанных тем, что всегда является замкнутой системой и содержит интегратор на входе. Интегратор взвешивает заряды выходного $U_{d}(N)$, смещающего $v=$ const и управляющего $U_{\text {y }}$ сигналов относительно заряда $Q$, сбрасывающего интегратор $\delta$-импульса. Указанный $\delta$-импульс появляется в начале каждого цикла интегрирования, а следующий управляющий импульс формируется в момент, когда интеграл всех указанных сигналов достигает нулевого значения [ $\left.{ }^{6}\right]$. Коммутационная функция OAC:

$$
\begin{aligned}
& L_{l}=0, \text { если }\left(N<N_{\alpha}\right) \bigvee\left(N>N_{\alpha+1}\right), \\
& L_{l}=1, \text { если } N_{\alpha}<N<N_{\alpha+1},
\end{aligned}
$$

где $N_{\alpha}$ и $N_{\alpha+1}$ определяются как корни системы интегральных уравнений

$$
Q-\int_{N_{\alpha-1}}^{N_{\alpha}}\left(U_{d}(N)+v-U_{\mathrm{y}}\right) d N=0
$$




$$
Q-\int_{N_{\alpha}}^{N}\left(U_{d}(N)+v-U_{\mathrm{y}}\right) d N=0
$$

Трансцендентность (13) указывает на то обстоятельство, что УВ с OAC работает в режиме динамического равновесия, при нарушении которого происходит процесс установления. Другими словами, если априори неизвестное из-за несимметричности сети значение $N_{\alpha}=N_{1}$ будет установлено с ошибкой относительно его равновесного значения, то нестационарная MM обусловит соответствующе искаженный спектр. Ошибками начальных условий $\left\{N_{\alpha}\right\}$ объясняется рассеивание расчетных для OAC данных, в связи с чем на рис. 6,2 мы можем представить лишь вероятную область изыскиваемой функциональной зависимости. Граф ММ УВ с ОАС показан на рис. 4. ММ этой системы ввиду ее специфичности дополнена ограничениями относительно минимально $\left(U_{\text {л+1 }} \geqslant U_{\text {л }}\right)$ и максимально $\left(U_{\text {л }}<-0,5\right)$ допустимых значений угла управления.

7. Многоканальная асинхронная СИФУ (МАС) из [7], MМ которой также представляет собой замкнутую систему, связывающую шесть интеграторов. Каждый интегратор взвешивает заряды управляющего сигнала $U_{\text {y }}$ за период $T$ сети $N=360$ и питающего выпрямитель напряжения вне интервала проводимости данного вентиля. Коммутационная функция МАC аналогична коммутационной функции ОАС (12), а $N_{\alpha}$ и $N_{\alpha+1}$ определяются как корни системы интегральных уравнений

$$
\begin{gathered}
\int_{N_{\alpha+1}}^{N_{\alpha}} U_{\text {Jl }}(N) d N-\int_{T} U_{\mathrm{y}}(t) d t=0, \\
\int_{N_{\alpha+2}}^{N_{\alpha+1}} U_{\pi l+1}(N) d N-\int_{T} U_{\mathrm{y}}(t) d t=0,
\end{gathered}
$$

И здесь система трансцендентных уравнений (14) характеризует динамическое равновесное состояние УВ с МАС. Поскольку (из-за большой длительности интегрирования) время установления МАС больше такового при ОАС и ожидаемая точность расчета гармонического состава низкая, то мы прибегли к решению уравнения (14) «вручную», предварительно преобразовав его в более удобную форму. В среднем за три шага итерации данная система установилась с точностью $\pm 5 \%$. Полученные фазовые соотношения (предварительный расчет велся в аналоговой форме) квазиустойчивого состояния МАC брались при машинном расчете за исходное множество начальных условий. Эта предварительная, хотя и трудоемкая, процедура позволила получить близкую к гладкой функциональную зависимость изыскиваемых соотношений. Граф ММ УВ с МАС показан на рис. 5.

По результатам цифрового анализа получена многомерная табличная функция первой гармонической составляющей (а также других неканонических и канонических, не входящих в тематику настоящей статьи): $U_{100} / U_{1}=f\left(\alpha \Leftrightarrow U_{y}, \varepsilon, \psi, \quad\right.$ СИФУ $) . \quad$ Графики $U_{100} / U_{1}=f\left(\alpha \Leftrightarrow U_{y}, \varepsilon\right)$ показаны на рис. $6, a-2$. Существенная зависимость $U_{100} / U_{1}=f(\psi)$, имеющая период $2 \pi / 3$, обнаружена лишь для MCE. На рис. $6, a$ она 


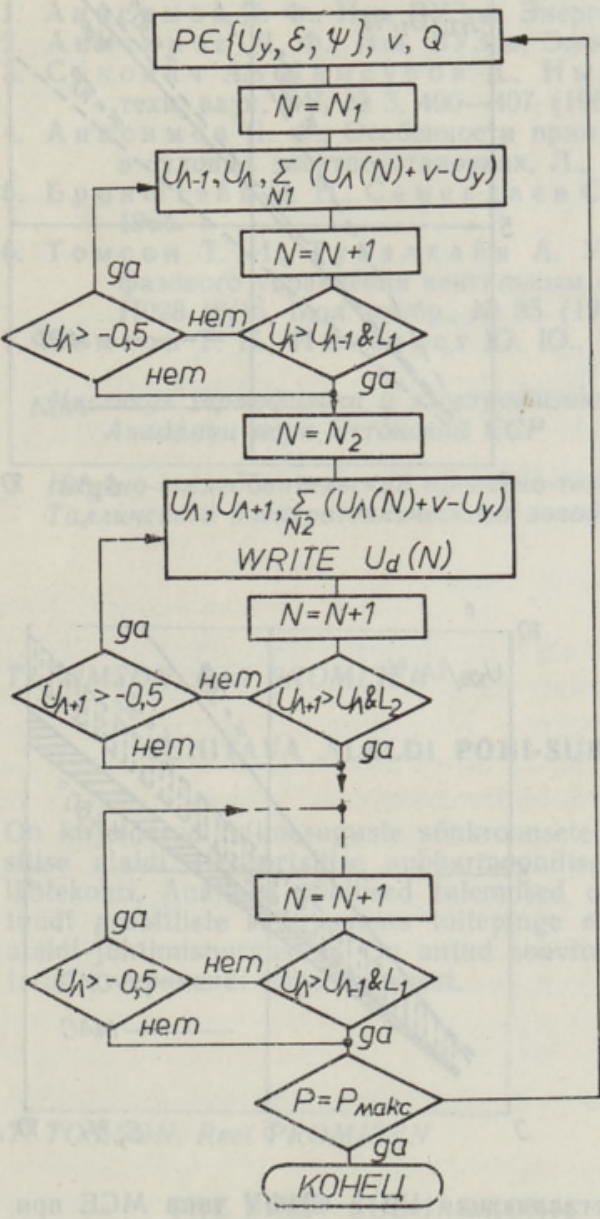

Рис. 4. ММ УВ, оснащенного одноканальной асинхронной СИФУ (ОАС).

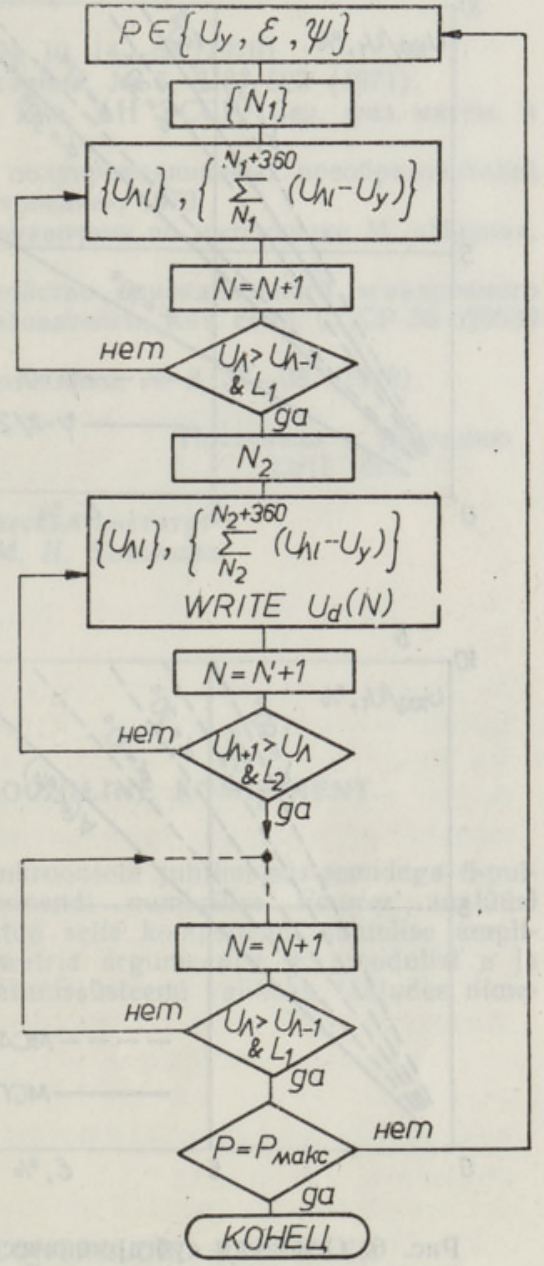

Рис. 5. ММ УВ, оснащенного многоканальной асинхронной СИФУ (MAC).

показана для двух экстремальных случаев. Поскольку эта зависимость для других СИФУ проявляется весьма слабо, результаты приведены лишь для $\psi=\pi / 3$ (рис. $6, \sigma-2$ ).

Анализ графиков позволяет сделать следующие выводы:

1. Амплитудная зависимость составляющей $100 \Gamma u$ от коэффициента несимметричности сети является монотонной функцией, близкой к линейной.

2. Эта зависимость, как правило, становится все значительнее с увеличением угла управления (исключения оговорены ниже).

3. СИФУ с равноинтервальным алгоритмом (МСП) инвариантна к $(\alpha, \psi)$; этот результат полностью подтверждает теоретические выводы Я. Ф. Анисимова $\left[{ }^{4}\right]$.

4. В пределах точности анализа таким же свойством обладает и ОАС. 

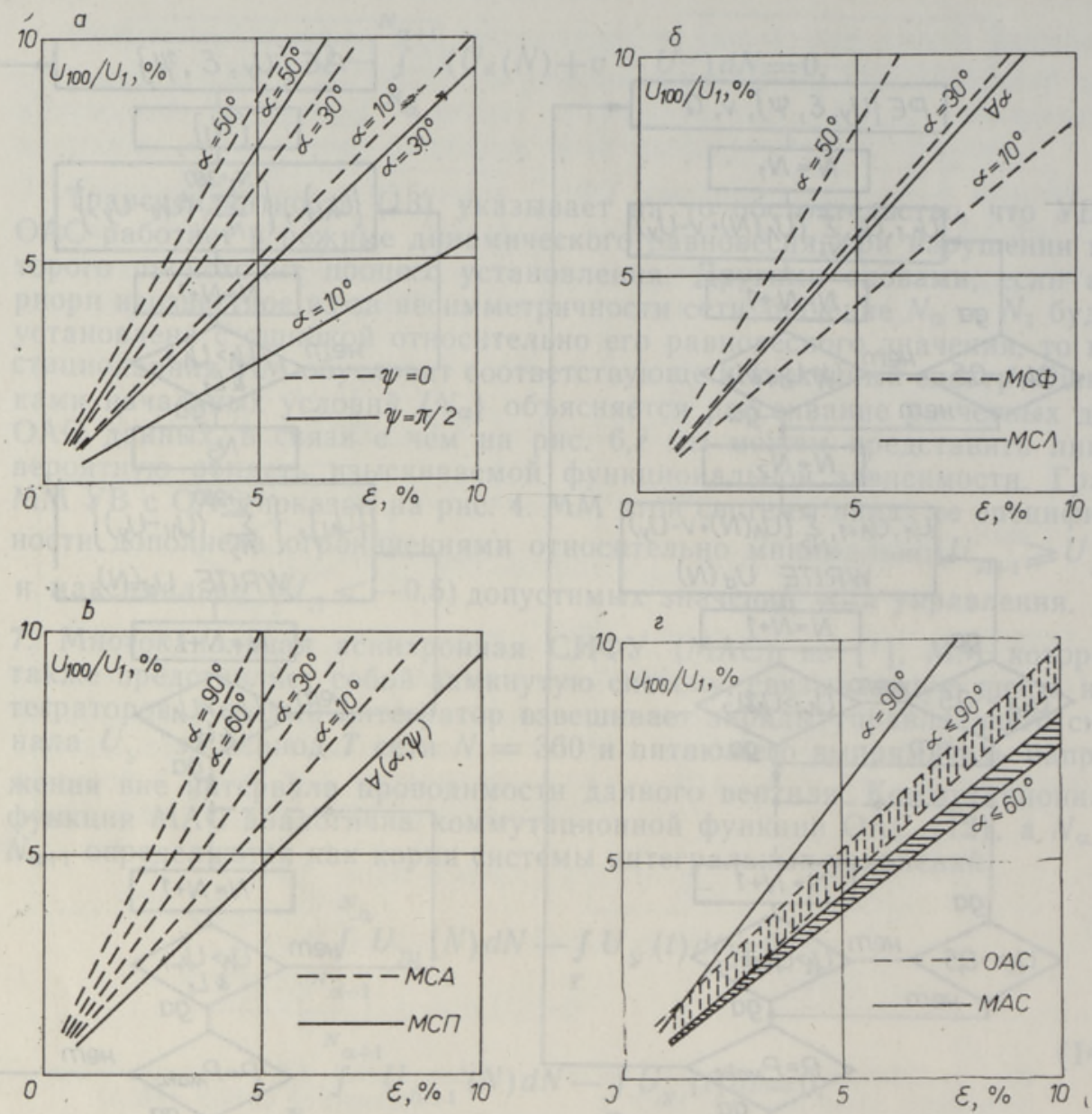

Рис. 6. Основная субгармоническая составляющая УВ с СИФУ типа МСЕ при вариации $\psi(a)$ и при $\psi=\pi / 3$ типов МСЛ и МСФ (б), типов МСП и МСА $(8)$, типов ОАС и МАС (2).

5. Качество СИФУ в смысле подавления составляющей $100 Г ц$ определяется частной производной $\partial U_{100} / \partial \varepsilon$, которую следует выбирать с минимальным значением.

6. Среди известных технических решений СИФУ наилучшей в указанном смысле системы не существует. Минимизация составляющей $100 \mathrm{\Gamma u}$ требует СИФУ с адаптирующим алгоритмом работы, управляемой по аргументу несимметричности сети. В этом плане могут оказаться перспективными СИФУ на базе микро-ЭВМ.

7. Среди рассмотренных систем предпочтение следует отдавать СИФУ типа МАС, если работа УВ в режиме глубокого регулирования $\neg(\alpha \rightarrow \pi / 2)$ исключена. В случае глубокого регулирования следует применять СИФУ типа МСП или ОАС.

Авторы выражают благодарность К. Кыннусаару за оказанную им помощь при наладке программ и обсуждении результатов. 


\title{
Л И Т Е Р А Т У Р А
}

1. Ан и си м ов Я. Ф., Изв. ВУЗов, Энергетика, № 10, 14-18 (1970).

2. А н и и м ов Я. Ф., Изв. ВУЗов, Электромеханика, № 5, 518-523 (1971).

3. Сакович А., Мам суров А., Н в в А., Изв. АН ЭССР, Сер. физ.-матем. и техн. наук, XV, № 3, 400-407 (1966).

4. А н и с и мов Я. Ф., Особенности применения полупроводниковых преобразователей в судовых электроустановках, Л., «Судостроение», 1973.

5. Б ронш тей Н И. Н., С еменд а ев С. А., Справочник по математике, М., «Наука», 1964.

6. Томсон Т. И., Т убалкайн А. У., Устройство одноканального асинхронного фазового управления вентильным преобразователем, Авт. свид. СССР № 529539 Н028 13/16, Бюл. изобр., № 35 (1976).

7. Томсон Т. И., Й ге в ест Ю. Ю., Электротехника, № 8, 37-38 (1978).
Ннститут термофизики и электрофизики Академии наук Эстонской ССР
Поступила в редакцию 23/II 1981

\begin{abstract}
Научно-исследовательский проектно-технологический институт
Таллинского электротехнического завода им. М. И. Калинина
\end{abstract}

\section{T. TOMSON, Reet PROMISEN}

\section{JUHITAVA ALALDI POHI-SUBHARMOONILINE KOMPONENT}

On kirjeldatud mitmesuguste sünkroonsete ja asünkroonsete juhtimissüsteemidega 6-pulsilise alaldi 100-hertsilise subharmoonilise komponendi numbrilise Fourier' analüüsi lähtekohti. Analüüsi pōhilised tulemused on esitatud selle komponendi suhtelise amplituudi graafiliste sõltuvustena toitepinge ebasümmeetria argumendist $\psi$, moodulist $\varepsilon$ ja alaldi juhtimisnurgast $\alpha$. On antud soovitused juhtimissüsteemi valikuks, lähtudes nimetatud komponendi vähendamisest.

\section{T. TOMSON, Reet PROMISEN}

\section{THE FIRST SUBHARMONIC OF A CONTROLLED RECTIFIER}

The influence of various control principles on the subharmonic composition of a 6-pulse thyristor rectifier is studied in this paper by the digital Fourier analysis. Every control circuit is considered by its commutation function $L_{l}$ in the mathematical model of the rectifier used as a subprogram in the basic Fourier analysis program. The results of the analyses are given by graphical functions in Fig. 6 as a part of the relative amplitude of the first subharmonic from the net asymmetry module $\varepsilon$. The following control circuits belong to the analysis;

1. Synchronous line-sweep control circuit MCE synchronized by the moment of «natural» commutation.

2. Synchronous line-sweep control circuit MСЛ synchronized by the zero-cross moment of line voltage.

3. Synchronous line-sweep control circuit MCФ synchronized by the zero-cross moment of phase voltage.

4. Synchronous line-sweep control circuit MCП synchronized by the zero-cross moment of a consent component $U_{1}$.

5. Synchronous sine-sweep control circuit MCA.

6. Single-channel asynchronous control circuit OAC.

7. Multi-channel asynchronous control circuit MAC.

No known optimal control circuit exists for a large variation of conditions, and minimizing the first subharmonic is possible only by an adaptive control algorithm. 